\title{
Entiers sans grand ni petit facteur premier II
}

\author{
par
}

\author{
ERIC SAIAS (Paris)
}

1. Introduction et énoncé des résultats. Notre objet dans ce présent travail est d'évaluer la quantité $\Theta(x, y, z)$ égale au nombre des entiers $\leq x$ et dont tous les facteurs premiers sont dans l'intervalle $] z, y]$. Pour bien comprendre cette étude, il est utile de rappeler certains résultats concernant les cas particuliers des fonctions de crible $\Psi(x, y)=\Theta(x, y, 1)$ et $\Phi(x, z)=$ $\Theta(x, x, z)$. Tout au long de cet article, on fera un usage systématique des notations suivantes :

$$
u=\frac{\log x}{\log y}, \quad v=\frac{\log x}{\log z} \quad \text { et } \quad r=\frac{u}{v}=\frac{\log z}{\log y} .
$$

Les meilleures approximations régulières des fonctions $\Psi(x, y)$ (pour $u$ petit) et $\Phi(x, z)$ sont des convolées d'une certaine solution d'équation différentielle aux différences avec une mesure appropriée. Ainsi, nous avons démontré dans [14] que la formule

$$
\Psi(x, y)=\Lambda(x, y)\left(1+O_{\varepsilon}\left(L_{\varepsilon}(y)^{-1}\right)\right)
$$

était valable dans le domaine

$$
\left(\mathrm{H}_{\varepsilon}(x, y)\right) \quad x \geq x_{0}, \quad x \geq y \geq \exp \left\{(\log \log x)^{5 / 3+\varepsilon}\right\}
$$

avec

$$
\Lambda(x, y)=x \int_{0}^{\infty} \varrho(u-t) d\left(\frac{\left[y^{t}\right]}{y^{t}}\right)
$$

et

$$
L_{\varepsilon}(y)=\exp \left\{(\log y)^{3 / 5-\varepsilon}\right\}
$$

(on se rapportera à [15] pour les définitions précises des trois solutions d'équation différentielle aux différences que sont les fonctions $\varrho$ de Dickman, $\omega$ de Buchstab et $\sigma$ de Friedlander). Par ailleurs, Tenenbaum ([20], Théorème III 6.7) a montré que pour $x \geq z \geq 2$, on a

$$
\Phi(x, z)=M(x, z)+O_{\varepsilon}(\Psi(x, z) E(x, z))
$$


avec

$$
M(x, z)=x e^{\gamma} \log z \prod_{p \leq z}\left(1-\frac{1}{p}\right) \int_{0}^{\infty} \omega(v-t) z^{-t} d t
$$

et

$$
E(x, z)=\left\{\begin{array}{cc}
L_{\varepsilon}(z)^{-1} \exp \left\{-c \frac{v}{\log ^{2}(v+1)}\right\}+ & \exp \left\{-(\log z)^{3 / 2-\varepsilon}\right\} \\
& \left(\text { dans le domaine } \mathrm{H}_{\varepsilon}(x, z)\right), \\
1 & \text { (hors de } \left.\mathrm{H}_{\varepsilon}(x, z)\right) .
\end{array}\right.
$$

La lettre $\gamma$ désigne la constante d'Euler et la lettre $c$ une constante positive convenable. Ces résultats améliorent les estimations de de Bruijn ([2], (1.3) et [1], (1.7)) qui a été le premier à introduire les fonctions $\Lambda(x, y)$ et $M(x, z)$ dans ce contexte. L'approximation de fonctions de crible par des fonctions convolées d'une solution d'une équation différentielle aux différences avec une mesure adéquate est en fait un phénomène beaucoup plus général décrit par Levin et Faŭnleı̆b ([13], voir aussi par exemple [5]). Pour le cas spécifique qui nous intéresse ici, on a deux fonctions qui apparaissent comme des candidats naturels à l'approximation de $\Theta$, suivant que l'on cherche à généraliser les formules (1.1) ou (1.2). Par l'identité du crible combinatoire, on a

$$
\Theta(x, y, z)=\sum_{P^{+}(d) \leq z} \mu(d) \Psi\left(\frac{x}{d}, y\right)
$$

où $P^{+}(d)$ désigne le plus grand facteur premier de $d$ et $\mu$ la fonction de Möbius. Au vu de (1.1), la quantité suivante est donc une approximation naturelle de $\Theta(x, y, z)$ :

$$
\Lambda(x, y, z)=\sum_{P^{+}(d) \leq z} \mu(d) \Lambda\left(\frac{x}{d}, y\right)=x \int_{0}^{\infty} \varrho(u-t) d\left(\frac{\Phi\left(y^{t}, z\right)}{y^{t}}\right) .
$$

D'autre part, on a aussi la quantité obtenue en substituant dans la dernière expression $\Phi\left(y^{t}, z\right)$ par $M\left(y^{t}, z\right)$, soit essentiellement

$$
M(x, y, z)=x \prod_{z<p \leq y}\left(1-\frac{1}{p}\right)^{-1} \int_{0}^{\infty} \sigma_{r}(u-t) y^{-t} d t
$$

où on a posé $\sigma_{r}(t)=\sigma(t, t / r)$.

Dans [5], Fouvry et Tenenbaum étudient la répartition des entiers sans grand facteur premier en progressions arithmétiques. En reprenant la démonstration de leur Théorème 2 dans le cas particulier qui nous intéresse ici, on obtient le résultat suivant. 
ThÉORÈme 0 (Fouvry et Tenenbaum). Soit $\varepsilon>0$. Sous les conditions $\mathrm{H}_{\varepsilon}(x, y)$ et $y \geq z^{2+\log (u+1)} \geq 1$, on a

$$
\Theta(x, y, z)=\Lambda(x, y, z)\left(1+O_{\varepsilon}\left(L_{\varepsilon}(y)^{-1}\right)\right) .
$$

Pour notre part, nous nous sommes intéressés à l'approximation de $\Theta(x, y, z)$ par $M(x, y, z)$. En utilisant une variante de la méthode du col (appelée encore méthode du point-selle) de manière analogue à ce que nous avons fait dans [14] pour démontrer la formule (1.1) dans le domaine $\left(\mathrm{H}_{\varepsilon}(x, y)\right)$, nous obtenons le résultat suivant :

THÉORÈme 1. Il existe une constante $c>0$ telle que pour tout $\varepsilon>0$, sous les conditions

$$
x \geq 3, \quad \exp \left\{(\log \log x)^{5 / 3+\varepsilon}\right\} \leq y \leq x
$$

et

$$
z \geq 3 / 2, \quad y \geq z^{1+c \sqrt{(\log 2 u) / u}} \geq 1,
$$

on ait

$$
\Theta(x, y, z)=M(x, y, z)\left(1+O_{\varepsilon}\left(\frac{\log 2 u}{(\log y) L_{\varepsilon}(z)}\right)\right) .
$$

$\mathrm{Au}$ vu des définitions de $\Lambda(x, y, z)$ et de $M(x, y, z)$, il est naturel de conjecturer que $\Lambda(x, y, z)$ est une meilleure approximation de $\Theta(x, y, z)$ que $M(x, y, z)$. Cependant les conditions $\left(\mathrm{H}_{\varepsilon}\right)$ et $\left(\mathrm{G}_{c}\right)$ sont bien moins restrictives que celles du Théorème 0 . Le problème de savoir si le Théorème 0 est encore valable en remplaçant la condition $y \geq z^{2+\log (u+1)} \geq 1$ par $\left(\mathrm{G}_{c}\right)$ reste pour l'instant ouvert.

Le Théorème 1 s'applique aux cas particuliers des fonctions $\Psi(x, y)$ et $\Phi(x, z)$. Sous la condition $\left(\mathrm{H}_{\varepsilon}\right)$, on a (cf. [15], Théorème 3(i))

$$
M(x, y, 1)=x \varrho(u)\left(1+O\left(\frac{\log 2 u}{\log y}\right)\right) .
$$

On obtient donc le résultat suivant, démontré initialement par Hildebrand avec une méthode d'équation fonctionnelle [8].

Corollaire 1 (Hildebrand). Soit $\varepsilon>0$. Sous la condition $\left(\mathrm{H}_{\varepsilon}\right)$, on a

$$
\Psi(x, y)=x \varrho(u)\left(1+O_{\varepsilon}\left(\frac{\log 2 u}{\log y}\right)\right) .
$$

Comme $M(x, x, z)=M(x, z)\left(1+O_{\varepsilon}\left(L_{\varepsilon}(x)^{-1}\right)\right)$, on obtient également le résultat suivant, démontré par de Bruijn $([1],(1.7))$ dans le domaine $\mathrm{H}_{\varepsilon}(x, z)$ et par Hildebrand et Maier ([10], Lemma 2) sous une forme légèrement différente, hors de $\mathrm{H}_{\varepsilon}(x, z)$. 
Corollaire 2 (de Bruijn, Hildebrand et Maier). Soit $\varepsilon>0$. Il existe une constante $z_{0}(\varepsilon)$ telle que sous la condition

$$
z_{0}(\varepsilon) \leq z \leq x^{1-\varepsilon}
$$

on ait

$$
\Phi(x, z)=M(x, z)\left(1+O_{\varepsilon}\left(\frac{1}{(\log x) L_{\varepsilon}(z)}\right)\right) .
$$

Il est à noter que ce résultat est plus faible que celui de Tenenbaum (formule (1.2)) cité ci-dessus.

La condition $\left(\mathrm{G}_{c}\right)$ du Théorème 1 impose apparemment au Corollaire 2 une condition du type $z \leq x^{c}$ avec $c$ convenable, plutôt qu'une condition $z \leq x^{1-\varepsilon}$ comme annoncé. En fait, une lecture attentive de la démonstration du Théorème 1 montre que l'on peut adjoindre à la région $\left(\mathrm{G}_{c}\right)$ les triplets $(x, x, z)$ vérifiant la condition (1.5), sans autre modification de l'énoncé.

$\mathrm{Au}$ vu des Corollaires 1 et 2, on voit que le Théorème 1 fournit toute une famille d'estimations asymptotiques qui relie continûment la formule d'Hildebrand (1.4) à celle de de Bruijn (1.6). Notre travail apparaît donc comme une unification des théories d'approximations des fonctions $\Psi(x, y)$ et $\Phi(x, z)$. Cela illustre une fois de plus la force, la pertinence et la simplicité conceptuelle de la méthode du point-selle dans ce type de problème.

Cette méthode avait pratiquement disparu de la littérature arithmétique pendant ces dernières décennies. Elle est réapparue de manière spectaculaire dans les travaux de Tenenbaum et Hildebrand ([18] et [11]). Depuis, elle est redevenue un outil central en théorie analytique des nombres (voir [9], [12], [14], [4], [7] et [17]). Nous renvoyons le lecteur à [19] pour un aperçu général de l'utilisation de la méthode du col en théorie des nombres.

Revenons au processus d'unification des théories de $\Psi$ et de $\Phi$ évoqué plus haut. C'est une des caractéristiques de la méthode du col que de permettre de démontrer dans une même démarche des résultats de nature assez, voire très différentes les uns des autres. C'est en particulier le cas dans [11] où Hildebrand et Tenenbaum font une étude unifiée de $\Psi(x, y)$ dans tout le domaine $x \geq y \geq 2$. Or on sait que le comportement asymptotique de $\Psi(x, y)$ diffère fondamentalement suivant que $y$ est grand ou petit (relativement à $x$ ). Cependant, il est à noter que cette capacité d'unification de la méthode du point-selle trouve sa contrepartie au niveau des calculs, où l'on doit sans cesse discuter suivant les valeurs relatives des paramètres.

On peut donner de multiples formules d'approximation de $M(x, y, z)$ combinant à des degrés divers simplicité et précision, ce qui fournit par l'intermédiaire du Théorème 1 autant de formules d'approximations de $\Theta(x, y, z)$. Nous en choisissons cinq que nous réunissons dans l'énoncé suivant : 
ThÉORÈme 2. Il existe une constante $c>0$ telle que pour tout $\varepsilon>0$, sous les conditions $\left(\mathrm{H}_{\varepsilon}\right)$ et $\left(\mathrm{G}_{c}\right)$, on ait

$$
\Theta(x, y, z)=\frac{x}{\log z} \exp \left\{-u \log u-u \log \left(\log u+\frac{\log y}{\log (y / z)}\right)+O_{\varepsilon}(u)\right\},
$$

(ii) (1.7)

$$
\begin{aligned}
& \left(1-\frac{\xi_{r}(u)}{\log y}\right) \Theta(x, y, z)=x \prod_{p \leq z}\left(1-\frac{1}{p}\right) \sqrt{\frac{\xi_{r}^{\prime}(u)}{2 \pi}} \\
& \quad \times \exp \left\{\gamma-\int_{1-r}^{u} \xi_{r}(t) d t\right\}\left(1+O_{\varepsilon}\left(\frac{\log u}{(\log y) L_{\varepsilon}(z)}+\frac{1}{u}\right)\right),
\end{aligned}
$$

$$
\Theta(x, y, z)=e^{\gamma} \prod_{p \leq z}\left(1-\frac{1}{p}\right)\left(x \sigma(u, v)+\frac{\log z}{\log y} y\right)\left(1+O_{\varepsilon}\left(\frac{\log 2 u}{\log y}\right)\right) .
$$

(iv) Il existe une constante $c>0$ telle que pour tout $\varepsilon>0$, sous les conditions $\left(\mathrm{H}_{\varepsilon}\right),\left(\mathrm{G}_{c}\right)$ et $x \geq y^{1+\varepsilon}$, on ait

$$
\Theta(x, y, z)=\frac{x \sigma(u, v)}{\log z}\left(1+O_{\varepsilon}\left(\frac{\log u}{\log y}+\frac{1}{L_{\varepsilon}(z)}\right)\right) .
$$

(v) Pour tout entier $n \geq 1$, il existe une constante $c_{n}>0$ telle que pour tout $\varepsilon>0$, sous les conditions $\left(\mathrm{H}_{\varepsilon}\right),\left(\mathrm{G}_{c_{n}}\right)$ et $x \geq y^{n+1+\varepsilon}$, on ait

$$
\begin{aligned}
\Theta(x, y, z)=e^{\gamma} x & \prod_{p \leq z}\left(1-\frac{1}{p}\right)\left[\sum_{k=0}^{n} \frac{(-1)^{k} \sigma_{r}^{(k)}(u)}{(\log y)^{k}}\right. \\
& \left.+O_{n, \varepsilon}\left(\sigma_{r}(u)\left(\left(\frac{\log u}{\log y}\right)^{n+1}+\frac{\log u}{(\log y) L_{\varepsilon}(z)}\right)\right)\right] .
\end{aligned}
$$

Le point (i) constitue une généralisation partielle de l'estimation suivante qui découle du travail de Canfield, Erdős et Pomerance (cf. Corollary de [3]).

Soit $\varepsilon>0$. Sous la condition $y \geq 2,(\log x)^{1+\varepsilon} \leq y \leq x$, on a

$$
\Psi(x, y)=x \exp \{-u \log u-u \log \log 2 u+O(u)\} .
$$

Ce résultat découle aussi naturellement des résultats de Hildebrand et Tenenbaum [11] sur $\Psi$. En reprenant la démarche de ces derniers dans le cas général de $\Theta$, nous démontrons au Théorème 4(ii) de [16] que la formule (i) est en fait valable sous les conditions $\log x \leq y-z$ et $\left(\mathrm{G}_{c}\right)$.

La formule (iii), qui apparaît dans [15] sous l'appellation du Théorème A, réalise à notre sens un bon compromis entre simplicité du terme principal, qualité du terme reste et extension du domaine de validité (on n'a pas besoin d'ajouter une condition supplémentaire du type $x \geq y^{1+c}$ comme pour (iv) et $(\mathrm{v}))$. On retrouve sous une forme plus simple et légèrement affaiblie la famille d'estimation de $\Theta(x, y, z)$ qui relie continûment l'approximation de 
$\Psi(x, y)$ qu'est la formule de Hildebrand (Corollaire 1) à une estimation de $\Phi(x, z)$ dans le domaine (1.5) qui est ici

$$
\Phi(x, z)=e^{\gamma} \omega(v) x \prod_{p \leq z}\left(1-\frac{1}{p}\right)\left(1+O_{\varepsilon}\left(\frac{1}{\log x}\right)\right) .
$$

Rappelons que Friedlander ([6], Theorem 1) démontre une formule essentiellement équivalente à (1.8) dans le domaine $1+\varepsilon \leq u \leq v \ll_{\varepsilon} 1$. Le point (iv) réalise donc une large extension du domaine de validité de la formule de Friedlander (1.8).

La formule (1.8) est apparentée à de nombreux développements asymptotiques de même nature qui apparaissent dans la littérature (voir par exemple le Corollaire 2 et les Théorèmes 8 et 9 de [5]).

Le point (iii) correspond au cas $n=0$ du point (v).

Démonstration du Théor ème 1 . Nous déduisons le Théorème 1 du résultat plus général qu'est le Théorème 3 énoncé ci-dessous. Conformément aux notations de [15], pour $0 \leq r<1$ et $u>1-r$ on désigne par $\xi_{r}(u)$ l'unique solution positive de l'équation

$$
e^{\xi_{r}(u)}=e^{r \xi_{r}(u)}+u \xi_{r}(u) .
$$

On pose en outre $\xi_{r}(1-r)=0$. De plus, on note $I_{r}(s)$ la fonction entière

$$
I_{r}(s)=\int_{0}^{s} \frac{e^{w}-e^{r w}}{w} d w .
$$

Enfin on désigne par $\left(\mathrm{V}_{\varepsilon}\right)$ le domaine suivant :

$$
z \geq 1, \quad y \geq z\left(1+L_{\varepsilon}^{-1}(z)\right) \text {. }
$$

ThÉORÈme 3. Soit $\varepsilon>0$. Sous les conditions $\left(\mathrm{H}_{\varepsilon}\right)$ et $\left(\mathrm{V}_{\varepsilon}\right)$, on a

$$
\Theta(x, y, z)=M(x, y, z)+O_{\varepsilon}\left(x \frac{\exp \left\{-u \xi_{r}(u)+I_{r}\left(\xi_{r}(u)\right)\right\} \log 2 u}{\sqrt{u}(\log y) L_{\varepsilon}(z)}\right) .
$$

Le terme d'erreur peut être rendu plus explicite par l'intermédiaire de la formule suivante (cf. Lemme 1 et Lemme 2(i))

$$
-u \xi_{r}(u)+I_{r}\left(\xi_{r}(u)\right)=-u\left[\log \left(u\left(\frac{1}{1-r}+\log 2 u\right)\right)+O(1)\right] .
$$

A titre de comparaison, il résulte du Theorem 3.2.2 de Levin et Faŭnleĭb ([13]) que pour $x \geq y \geq z \geq 1$, on a

$$
\Theta(x, y, z)=M(x, y, z)+O_{\varepsilon}\left(\frac{x(\log x)^{A}}{L_{\varepsilon}(z)}\right)
$$

où $A$ désigne une constante positive convenable. Il est à noter que notre méthode permet également de majorer $\Theta(x, y, z)-M(x, y, z)$ hors de $\left(\mathrm{V}_{\varepsilon}\right)$. 
On obtient que pour $x, y$ et $z$ vérifiant $\left(\mathrm{H}_{\varepsilon}\right)$ mais pas $\left(\mathrm{V}_{\varepsilon}\right)$, on a

$$
\Theta(x, y, z)=M(x, y, z)+O_{\varepsilon}\left(x \exp \left\{-u(\log y)^{3 / 5-\varepsilon}\right\}\right) .
$$

Mais ce résultat est alors inférieur à celui obtenu facilement par la méthode de Rankin, soit

$$
\begin{aligned}
\Theta(x, y, z) & \leq \sum_{z<P^{-}(n) \leq P^{+}(n) \leq y}\left(\frac{x}{n}\right)^{\beta} \\
& =x^{\beta} \prod_{z<p \leq y}\left(1-p^{-\beta}\right)^{-1} \asymp x \frac{\log y}{\log z} \exp \left\{-u \xi_{r}(u)+I_{r}\left(\xi_{r}(u)\right)\right\}
\end{aligned}
$$

d'après (5.6), où on a posé $\beta=1-\xi_{r}(u) / \log y$.

Le Théorème 1 résulte immédiatement du Théorème 3 et de l'estimation de $M(x, y, z)$ suivante.

THÉORÈme 4 . Il existe une constante $c>0$ telle que sous les conditions $\left(\mathrm{G}_{c}\right)$ et

$$
x \geq y \geq(\log x)^{2},
$$

on ait

$$
M(x, y, z) \asymp x \frac{\exp \left\{-u \xi_{r}(u)+I_{r}\left(\xi_{r}(u)\right)\right\}}{\sqrt{u} \log z} .
$$

Nous achevons cette introduction avec la version conditionnelle de nos résultats. Sous l'hypothèse de Riemann, on peut remplacer dans les énoncés des Théorèmes 1,2 et 3 le domaine $\left(\mathrm{H}_{\varepsilon}\right)$ par le domaine

$$
z \geq 3 / 2, \quad x \geq y \geq 2, \quad \log x \leq(y-z) / y^{1 / 2+2 \varepsilon}
$$

et supprimer la condition $\left(\mathrm{V}_{\varepsilon}\right)$, tout cela à la condition de remplacer les estimations (1.3) et (1.7)-(1.10) par les formules suivantes :

$$
\begin{gathered}
\Theta(x, y, z)=M(x, y, z)\left(1+O_{\varepsilon}\left(\frac{(\log 2 u) y}{(\log y)(y-z) z^{1 / 2}-(\log u) / \log y-\varepsilon}\right)\right) \\
\left(1-\frac{\xi_{r}(u)}{\log y}\right) \Theta(x, y, z)=x \prod_{p \leq z}\left(1-\frac{1}{p}\right) \sqrt{\frac{\xi_{r}^{\prime}(u)}{2 \pi}} \exp \left\{\gamma-\int_{1-r}^{u} \xi_{r}(t) d t\right\} \\
\times\left(1+O_{\varepsilon}\left(\frac{(\log u) y}{\left.\left.(\log y)(y-z) z^{1 / 2-(\log u) / \log y-\varepsilon}+\frac{1}{u}\right)\right)}\right.\right. \\
\Theta(x, y, z)=\frac{x \sigma(u, v)}{\log z}\left(1+O_{\varepsilon}\left(\frac{\log u}{\log y}+\frac{1}{z^{1 / 2}-\varepsilon}\right)\right) \\
\Theta(x, y, z)=e^{\gamma} x \prod_{p \leq z}\left(1-\frac{1}{p}\right)\left[\sum_{k=0}^{n} \frac{(-1)^{k} \sigma_{r}^{(k)}(u)}{(\log y)^{k}}\right. \\
\left.+O_{n, \varepsilon}\left(\sigma_{r}(u)\left(\left(\frac{\log u}{\log y}\right)^{n+1}+\frac{\log u}{(\log y) z^{1 / 2-\varepsilon}}\right)\right)\right]
\end{gathered}
$$




$$
\begin{aligned}
\Theta(x, y, z)= & M(x, y, z)+O_{\varepsilon}\left(\frac{y}{(\log y)(y-z) z^{1 / 2-(\log u) / \log y-\varepsilon}}\right. \\
& \left.\times\left(\frac{\exp \left\{-u \xi_{r}(u)+I_{r}\left(\xi_{r}(u)\right)\right\} \log 2 u}{\sqrt{u}}+M(x, y, z)\right)\right) .
\end{aligned}
$$

2. Notations. Nous faisons ici la liste des principales notations employées dans cet article.

Pour la définition de la fonction de Friedlander $\sigma_{r}(u)=\sigma(u, v)$, nous renvoyons à [15]. La lettre $p$ désigne un nombre premier générique. Pour tout entier $n>1$, on désigne par $P^{+}(n)$ (respectivement $P^{-}(n)$ ) le plus grand (resp. petit) facteur premier de $n$. On pose de plus $P^{+}(1)=1$ et $P^{-}(1)=+\infty$. On a ainsi

$$
\Theta(x, y, z)=\sum_{n \leq x, P^{+}(n) \leq y, P^{-}(n)>z} 1 .
$$

La lettre $s$ désigne un nombre complexe, les réels $\kappa$ et $\tau$ étant définis implicitement par $s=\kappa+i \tau$.

Pour tout couple $(r, u)$ vérifiant $0 \leq r<1$ et $u>1-r$, on désigne par $\xi_{r}(u)$ l'unique solution positive de l'équation

$$
e^{\xi_{r}(u)}=e^{r \xi_{r}(u)}+u \xi_{r}(u) .
$$

On pose en outre $\xi_{r}(1-r)=0$. Pour simplifier l'écriture, on notera souvent $\xi$ pour $\xi_{r}(u)$. Pour tout réel $r$ vérifiant $0 \leq r<1$, on note $I_{r}(s)$ la fonction entière

$$
I_{r}(s)=\int_{0}^{s} \frac{e^{w}-e^{r w}}{w} d w
$$

On remarque que l'on a

$$
I_{r}^{\prime}\left(\xi_{r}(u)\right)=u .
$$

Pour Re $s>0$ et $y>z \geq 1$, on pose

et

$$
\zeta(s, y)=\prod_{p \leq y}\left(1-p^{-s}\right)^{-1}
$$

$$
\zeta(s, y, z)=\frac{\zeta(s, y)}{\zeta(s, z)}=\prod_{z<p \leq y}\left(1-p^{-s}\right)^{-1}=\sum_{P^{-}(n)>z, P^{+}(n) \leq y} n^{-s} .
$$

On notera $\xi^{\prime}(s, y, z)$ et $\xi^{\prime \prime}(s, y, z)$ pour $\frac{\partial}{\partial s} \zeta(s, y, z)$ et $\frac{\partial^{2}}{\partial s^{2}} \zeta(s, y, z)$.

On pose

$$
F(s, z)=\exp \left\{\int_{1}^{s}\left(\frac{\zeta^{\prime}(w)}{\zeta(w)}-\frac{\zeta^{\prime}(w, z)}{\zeta(w, z)}-\frac{z^{1-w}}{1-w}\right) d w\right\}-1
$$

où $\zeta(w)$ désigne la fonction zêta de Riemann. 
On définit la transformée de Laplace d'une fonction $f$ par

$$
\widehat{f}(s)=\int_{-\infty}^{\infty} f(t) e^{-s t} d t .
$$

Pour $t \geq 1$ et $0<\varepsilon<3 / 5$, on note

$$
L_{\varepsilon}(t)=\exp \left\{(\log t)^{3 / 5-\varepsilon}\right\} .
$$

Pour $x \geq y>z \geq 1$ on note

$$
u=\frac{\log x}{\log y}, \quad v=\frac{\log x}{\log z} \quad \text { et } \quad r=\frac{u}{v}=\frac{\log z}{\log y} .
$$

En particulier si $z=1$ on a $v=+\infty$ et $r=0$.

On pose

$$
\beta=1-\frac{\xi_{r}(u)}{\log y} .
$$

On désigne par $\gamma$ la constante d'Euler.

Toutes les constantes implicites dans l'utilisation des symboles $\asymp, O$ de Landau et $\ll$ de Vinogradov sont absolues sauf indication de dépendance mentionnée en indice.

La lettre $c$ désigne tout au long de cet article une constante positive convenable, non nécessairement la même à chaque occurrence. En particulier, à chaque fois que l'on écrit "Sous la condition $\left(\mathrm{G}_{c}\right), \ldots$ ", on doit comprendre "il existe une constante $c>0$ telle que sous la condition $\left(\mathrm{G}_{c}\right), \ldots$ "

\section{Démonstrations des Théorèmes 2 et 4}

Démonstration du Théorème 2. Compte tenu de l'étude des fonctions $M(x, y, z)$ et $\sigma(u, v)$ effectuée dans [15], il est pratiquement immédiat de déduire le Théorème 2 du Théorème 1 et d'établir le Théorème 4.

Voyons cela en détail. Montrons tout d'abord que pour tout réel $u_{0}$, il existe une constante $c>0$ telle que

$$
\xi_{r}(u) \ll 1 \asymp \sigma(u, v) \ll \xi_{r}^{\prime}(u) \quad\left(1 \leq u \leq u_{0},\left(\mathrm{G}_{c}\right)\right) .
$$

En reprenant les calculs effectués pour démontrer les points (iv), (v) et (vi) du Lemme 4 de [15], on montre facilement que pour $1 \leq u \leq u_{0}$, on a $\xi_{r}(u) \ll 1$. La minoration $\xi_{r}^{\prime}(u) \gg 1$ résulte alors de la formule (23) de [15]. Enfin, pour $c$ convenablement choisi on a $\sigma(u, v) \asymp \varrho(u) \asymp 1$ d'après le Corollaire de [15] et les propriétés de $\varrho$ (cf. Théorème III 5.5 de [20]). Passons maintenant à la démonstration du Théorème 2 proprement dite.

On obtient le point (iii) du Théorème 2 en remplaçant dans le Théorème 1 la quantité $M(x, y, z)$ par son approximation qui apparaît au Théorème 3(i) de [15]. On obtient de même le point (v) en remplaçant $M(x, y, z)$ par son développement asymptotique (formule (12) de [15]). 
Si $u \ll 1$, on déduit (i), (ii) et (iv) de (iii) en utilisant (3.1) et la formule de Mertens. On suppose dorénavant $u$ suffisamment grand. On a alors d'après le Théorème 3(iii) de [15]

$$
M(x, y, z)=\left(1+O\left(\frac{1}{u}\right)\right) \prod_{z<p \leq y}\left(1-\frac{1}{p}\right)^{-1} \frac{x \sigma_{r}(u)}{\log y+\sigma_{r}^{\prime}(u) / \sigma_{r}(u)}
$$

avec par la forme forte de la formule de Mertens

$$
\prod_{p \leq y}\left(1-\frac{1}{p}\right)^{-1}=e^{\gamma} \log y\left(1+O_{\varepsilon}\left(L_{\varepsilon / 2}(y)^{-1}\right)\right),
$$

et, d'après le Théorème 1 de [15],

$$
\sigma_{r}(u)=\left(1+O\left(\frac{1}{u}\right)\right) \sqrt{\frac{\xi_{r}^{\prime}(u)}{2 \pi}} \exp \left\{\gamma-\int_{1-r}^{u} \xi_{r}(t) d t\right\}
$$

et

$$
\log y+\sigma_{r}^{\prime}(u) / \sigma_{r}(u)=\left(1+O\left(\frac{1}{u}\right)\right)(\log y)\left(1-\frac{\xi_{r}(u)}{\log y}\right) .
$$

Le point (ii) découle donc du Théorème 1.

Pour montrer le point (i), on a besoin de l'estimation suivante.

Lemme 1. Sous les conditions $0 \leq r<1$ et $u \geq 1$, on a

$$
\xi_{r}(u)=\log u+\log \left(\log 2 u+\frac{1}{1-r}\right)+O(1) .
$$

Démonstration. Cela résulte des points (iv), (v) et (vi) du Lemme 4 de $[15]$.

Revenons à la preuve du Théorème 2(i). D'après la formule (11) de [15], le Lemme 5(i) de [15] et le Lemme 1 ci-dessus, on a

$$
-\int_{1-r}^{u} \xi_{r}(t) d t=-u\left(\log u+\log \left(\log 2 u+\frac{1}{1-r}\right)+O(1)\right) .
$$

La formule de Mertens et l'estimation

$$
\xi_{r}^{\prime}(u) \asymp \frac{1}{u}
$$

(cf. Lemme 4(vii) de [15]) permettent de montrer que le point (i) découle du point (ii).

D'après (3.2), (3.3) et (3.4), on a

$$
y \ll_{\varepsilon} \frac{x \sigma(u, v) \log u}{\log z} \quad\left(y \geq(\log x)^{2},\left(\mathrm{G}_{c}\right) \text { et } x \geq y^{1+\varepsilon}\right) .
$$


A l'aide de la forme forte de la formule de Mertens, on voit donc que (iv) découle de (iii). Cela achève la démonstration du Théorème 2 .

Démonstration du Théorème 4. On a

$$
M(x, y, z) \asymp \frac{x \sigma_{r}(u)}{\log z} \quad\left(y \geq(\log x)^{2},\left(\mathrm{G}_{c}\right)\right) .
$$

En effet, si $y \ll 1$, les deux membres sont $\asymp 1$ d'après la définition de $M(x, y, z)$ et (3.1); et si $y$ est suffisamment grand, cela résulte du Théorème $3(\mathrm{i})$ de [15], de (3.5) et de (3.1).

D'après (3.1) pour $u$ borné, et (3.2), (3.4) et la formule (11) de [15] sinon, on a

$$
\sigma_{r}(u) \asymp \frac{\exp \left\{-u \xi_{r}(u)+I_{r}\left(\xi_{r}(u)\right)\right\}}{\sqrt{u}} .
$$

Cela permet de conclure la démonstration du Théorème 4 .

4. Schéma général de la démonstration du Théorème 3. Les deux quantités $\Theta(x, y, z)$, qui est de type arithmétique, et $M(x, y, z)$, qui est essentiellement de type analytique, s'expriment naturellement l'une et l'autre comme une intégrale sur une droite verticale du plan complexe. D'une part $\Theta(x, y, z)$ s'écrit sous la forme de son intégrale de Perron

$$
\Theta(x, y, z)=\frac{1}{2 i \pi} \int_{\beta-i \infty}^{\beta+i \infty} \frac{\zeta(s, y, z) x^{s}}{s} d s
$$

d'autre part $M(x, y, z)$ s'écrit par l'intermédiaire de la formule d'inversion de Laplace (voir Lemme 10)

$$
M(x, y, z)=\frac{x \zeta(1, y, z)}{2 i \pi} \int_{-\xi-i \infty}^{-\xi+i \infty} \frac{\exp \left\{I_{r}\left(-s^{\prime}\right)\right\}-r}{s^{\prime}+\log y} e^{u s^{\prime}} d s^{\prime} .
$$

Dans un premier temps, nous allons montrer (cf. Lemmes 7 et 8) que pour chacune des deux intégrales, la contribution de la partie correspondant à un voisinage convenable de l'infini du domaine d'intégration est négligeable.

Dans un deuxième temps et ce sera là le point crucial, sur le domaine borné restant, nous montrerons que les deux intégrandes sont proches l'une de l'autre (cf. Lemme fondamental (Lemme 4)), ce qui permettra d'établir que les deux intégrales correspondantes sont également voisines (cf. Lemme 9).

Nous résumons ce canevas de démonstration par le schéma suivant. 


$$
\begin{array}{cc}
\text { Arithmétique } & \text { Analyse } \\
\Theta(x, y, z) & M(x, y, z) \\
12 & 12 \\
\frac{1}{2 i \pi} \int_{\beta-i L_{\varepsilon}(z)}^{\beta+i L_{\varepsilon}(z)} \frac{\zeta(s, y, z) x^{s}}{s} d s \simeq \frac{x \zeta(1, y, z)}{2 i \pi} \int_{-\xi-i L_{\varepsilon}(z) \log y}^{-\xi+i L_{\varepsilon}(z) \log y} \frac{e^{I_{r}\left(-s^{\prime}\right)}-r}{s^{\prime}+\log y} e^{u s^{\prime}} d s^{\prime}
\end{array}
$$

On notera au passage l'utilisation du changement de variable $s^{\prime}=$ $(s-1) \log y$.

Le succès de cette entreprise tient au fait que dans les deux cas, on choisit comme abscisse d'intégration un nombre qui est, dans le domaine $\left(\mathrm{H}_{\varepsilon}\right)$, très proche du point-selle réel de l'intégrande. De manière précise, $-\xi$ est l'unique point-selle réel de la fonction $\exp \left\{I_{r}(-s)+u s\right\}$ (voir (2.1)).

5. Evaluation de $\exp \left\{I_{r}(s)\right\}$ et $\zeta(s, y, z)$. Le résultat suivant apparaît au Lemme 5 de [15].

Lemme 2. Soit $s=\xi_{r}(u)+i \tau$ avec $\tau \in \mathbb{R}$. Sous les conditions $0 \leq r<1$ et $u \geq 1$, on $a$

(iii) $\left|e^{I_{r}(s)}\right| \leq \exp \left\{I_{r}\left(\xi_{r}(u)\right)-c u \min ^{2}\left(1-r,(\log (u+2))^{-1}\right)\right\} \quad(|\tau|>\pi)$,

$$
\begin{gathered}
I_{r}\left(\xi_{r}(u)\right)=u\left(1+O\left(\frac{1}{\log (u /(1-r))}\right)\right), \\
\left|e^{I_{r}(s)}\right| \leq \exp \left\{I_{r}\left(\xi_{r}(u)\right)-c \tau^{2} u\right\} \quad(|\tau| \leq \pi), \\
e^{I_{r}(s)}=-\frac{e^{-\gamma}}{s}\left(1+O\left(\frac{e^{\xi}}{|\tau|}+|r \tau|\right)\right) \quad\left(e^{\xi} \leq|\tau| \ll r^{-1}\right), \\
e^{I_{r}(s)}=r\left(1+O\left(\frac{e^{\xi}}{|\tau|}+\frac{e^{r \xi}}{|r \tau|}\right)\right) \quad\left(|\tau| \geq e^{\xi}, r \tau \gg 1\right) .
\end{gathered}
$$

Pour le Lemme 3 ainsi que les suivants, on rappelle que, sauf indication explicite du contraire, les réels $\kappa$ et $\tau$ sont définis implicitement par la formule $s=\kappa+i \tau$.

Lemme 3. Soit $\varepsilon>0$. Il existe un $t_{0}=t_{0}(\varepsilon)$ tel que, sous les conditions

$$
t \geq t_{0}(\varepsilon), \quad 2 \geq \kappa \geq 1-(\log t)^{-2 / 5-\varepsilon} \quad \text { et } \quad|\tau| \leq L_{\varepsilon}(t),
$$

on ait

$$
\frac{\zeta^{\prime}(s, t)}{\zeta(s, t)}=\frac{\zeta^{\prime}(s)}{\zeta(s)}+\frac{t^{1-s}}{1-s}+O_{\varepsilon}\left(\frac{1}{L_{\varepsilon}(t)}\right)
$$

(ii) $\zeta(s, t)=\zeta(1, t)(s-1) \zeta(s) \exp \left\{I_{0}((1-s) \log t)\right\}\left(1+O_{\varepsilon}\left((s-1) / L_{\varepsilon}(t)\right)\right)$. 
Démonstration. Ce résultat apparaît au cours de la démonstration du Lemme III 5.9.1 du livre de Tenenbaum [20]. La formule du point (i) est équivalente à la formule III 5.71 de [20]. Le point (ii), que l'on retrouve sous une forme très légèrement plus faible à la formule III 5.67, s'obtient en intégrant (i) entre 1 et $s$.

Rappelons que l'on note

$$
F(s, z)=\exp \left\{\int_{1}^{s}\left(\frac{\zeta^{\prime}(w)}{\zeta(w)}-\frac{\zeta^{\prime}(w, z)}{\zeta(w, z)}+\frac{z^{1-w}}{1-w}\right) d w\right\}-1 .
$$

Lemme fondamental (ou Lemme 4). Soit $\varepsilon>0$. Il existe un $y_{0}=y_{0}(\varepsilon)$ tel que sous les conditions

$$
y \geq y_{0}(\varepsilon), \quad \kappa \geq 1-(\log y)^{-2 / 5-\varepsilon} \quad \text { et } \quad|\tau| \leq L_{\varepsilon}(y),
$$

on ait

$$
\begin{aligned}
& \zeta(s, y, z) \\
& =\zeta(1, y, z) \exp \left\{I_{r}((1-s) \log y)\right\}\left(1+F(s, z)+O_{\varepsilon}\left(\frac{1}{L_{\varepsilon}(y)}\right)\right) .
\end{aligned}
$$

De plus, sous les conditions $2 \geq \kappa \geq 1-(\log 3 z)^{-2 / 5-\varepsilon}$ et $|\tau| \leq L_{\varepsilon}(z)$, on a

$$
F(s, z) \ll_{\varepsilon} \frac{s-1}{L_{\varepsilon}(z)} .
$$

Démonstration. On a

$$
\frac{\zeta^{\prime}(s, y, z)}{\zeta(s, y, z)}=\frac{\zeta^{\prime}(s, y)}{\zeta(s, y)}-\frac{\zeta^{\prime}(s, z)}{\zeta(s, z)} .
$$

En appliquant le Lemme 3 (i) pour $t=y$, on obtient sous les conditions indiquées

$$
\frac{\zeta^{\prime}(s, y, z)}{\zeta(s, y, z)}=\frac{y^{1-s}-z^{1-s}}{1-s}+\frac{\zeta^{\prime}(s)}{\zeta(s)}-\frac{\zeta^{\prime}(s, z)}{\zeta(s, z)}+\frac{z^{1-s}}{1-s}+O_{\varepsilon}\left(\frac{1}{L_{\varepsilon / 2}(y)}\right) .
$$

On obtient alors (5.1) en intégrant entre 1 et $s$ cette dernière formule, puis en considérant la nouvelle égalité obtenue en prenant l'exponentielle des deux membres.

Si $z \geq z_{0}(\varepsilon)$, on obtient (5.2) en intégrant la formule du point (i) du Lemme 3 entre 1 et $s$. Si $z \leq z_{0}(\varepsilon)$, on a uniformément pour $w \in[1, z]$,

$$
\frac{\zeta^{\prime}(w)}{\zeta(w)}-\frac{\zeta^{\prime}(w, z)}{\zeta(w, z)}+\frac{z^{1-w}}{1-w} \ll 1
$$

et donc $F(s, z) \ll s-1$, ce qui permet de compléter la démonstration de (5.2). 
Les deux lemmes suivants permettront de majorer $\zeta(\beta+i \tau, y, z)$ dans la zone intermédiaire $L_{\varepsilon}(z) \leq|\tau| \leq L_{\varepsilon / 3}(y)$.

Lemme 5. Soit $\varepsilon>0$. Il existe un $y_{0}=y_{0}(\varepsilon)$ tel que sous les conditions

$$
y \geq y_{0}(\varepsilon), \quad \kappa \geq 1-(\log y)^{-2 / 5-\varepsilon} \quad \text { et } \quad \frac{y^{1-\kappa}}{\log y} \leq|\tau| \leq L_{\varepsilon}(y)
$$

on ait

$$
\zeta(s, y, z) \ll \log (|\tau|+2) \log z \cdot e^{I_{0}((1-\kappa) \log z)} .
$$

Lemme 6. Soit $\varepsilon>0$. On suppose vérifiées les conditions $\left(\mathrm{H}_{\varepsilon}\right),\left(\mathrm{V}_{\varepsilon}\right)$ et

$$
L_{\varepsilon}(z) \leq|\tau| \leq L_{\varepsilon / 3}(y) .
$$

Il existe alors un $y_{0}=y_{0}(\varepsilon)$ tel que

(i) sous les conditions supplémentaires $y \geq y_{0}(\varepsilon)$ et $r \geq 1 / 2$, on ait

$$
\zeta(\beta+i \tau, y, z)=1+O_{\varepsilon}\left(\frac{e^{\xi}}{|\tau \log y|}+\frac{1}{L_{\varepsilon}(z)}\right),
$$

(ii) sous les conditions supplémentaires $y \geq y_{0}(\varepsilon)$ et $r<1 / 2$, on ait

$$
\zeta(\beta+i \tau, y, z) \ll_{\varepsilon} \frac{\log y}{\log z} e^{I_{r}(\xi)-c u} .
$$

Démonstration du Lemme 5 . On estime $\zeta(s, y, z)=\zeta(s, y) /$ $\zeta(s, z)$ en majorant indépendamment $\zeta(s, y)$ et $\zeta(s, z)^{-1}$. Par le Lemme $3(\mathrm{ii})$, on a

$$
\zeta(s, y) \asymp_{\varepsilon} \tau(\log y) \zeta(s) \exp \left\{I_{0}((1-s) \log y)\right\} .
$$

En utilisant l'estimation de $I_{0}(s)$ du Lemme 7 de [14] et une majoration classique de $\zeta(s)$, on obtient $\zeta(s, y) \ll \log (|\tau|+2)$.

Par ailleurs on a

$$
\left|\zeta(s, z)^{-1}\right| \leq \zeta(\kappa, z) \ll \log z \cdot e^{I_{0}((1-\kappa) \log z)}
$$

d'après le point (ii) du Lemme 3 .

Démonstration du Lemme 6. Sous les conditions $\left(\mathrm{H}_{\varepsilon}\right),\left(\mathrm{V}_{\varepsilon}\right)$ et $|\tau| \geq L_{\varepsilon}(z)$, on a $|\tau| \geq e^{\xi}$. Pour (i) on peut donc appliquer le Lemme 4 en estimant $e^{I_{r}(\xi-i \tau \log y)}$ par le Lemme $2(\mathrm{v})$ et $\zeta(1, y, z)$ avec la formule de Mertens.

Pour (ii), notre premier objectif est de montrer que sous les conditions indiquées

$$
|\zeta(\beta+i \tau, y, z)| \ll \zeta(\beta, y, z) e^{-c u} .
$$

Pour cela, on peut supposer $u \geq u_{0}$ puisque l'on a toujours $\mid \zeta(\beta+$ $i \tau, y, z) \mid \leq \zeta(\beta, y, z)$. 
Nous appliquons alors la technique utilisée au Lemma 8 de [11]. On a

$$
|\zeta(\beta+i \tau, y, z)| \leq \zeta(\beta, y, z) e^{-w}
$$

avec

$$
w=\sum_{z<p \leq y}(1-\cos (\tau \log p)) p^{-\beta} .
$$

De plus

$$
\frac{1}{\log y} \sum_{z<n \leq y} \Lambda(n)(1-\cos (\tau \log n)) n^{-\beta} \leq w+2 S
$$

où $\Lambda$ désigne la fonction de von Mangoldt et

$$
S=\frac{1}{\log y} \sum_{\nu \geq 2} \sum_{p^{\nu} \leq y} p^{-\nu \beta} \log p .
$$

D'après le Lemme 1 , sous les conditions $y \geq(\log x)^{2+\varepsilon}$ et $r<1 / 2$, on a

$$
\beta \geq 1 / 2+O_{\varepsilon}(1 / \log y)
$$

et par suite $S \ll_{\varepsilon} 1$. En appliquant le Corollary du Lemma 6 de [11] pour estimer le membre de gauche de (5.5), on obtient donc

$$
\begin{aligned}
& w \geq \frac{1}{\xi}\left(e^{\xi}\left(1-\frac{1}{\sqrt{1+(\tau(\log y) / \xi)^{2}}}\right)-2 e^{r \xi}\right) \\
& +O\left(1+\exp \left\{\xi-(\log y)^{\varepsilon / 2}\right\}\right) \gg e^{\xi} / \xi \geq u .
\end{aligned}
$$

Avec (5.4), cela montre (5.3).

Pour conclure la démonstration du point (ii) du Lemme 6, il suffit maintenant de remarquer que d'après le Lemme 4 , on a

$$
\begin{aligned}
\zeta(\beta, y, z) & =\prod_{z<p \leq y}\left(1-p^{-\beta}\right)^{-1} \\
& =\frac{\log y}{\log z} e^{I_{r}(\xi)}\left(1+O_{\varepsilon}\left(\frac{1}{L_{\varepsilon}(z)}\right)\right) \quad\left(\left(\mathrm{H}_{\varepsilon}\right),\left(\mathrm{V}_{\varepsilon}\right)\right) .
\end{aligned}
$$

6. Démonstration du Théorème 3. Conformément au schéma du paragraphe 3, nous donnons les principales étapes de la démonstration du Théorème 3 sous la forme des trois lemmes suivants.

Lemme 7. Soit $\varepsilon>0$. Sous les conditions $\left(\mathrm{H}_{\varepsilon}\right)$ et $\left(\mathrm{V}_{\varepsilon}\right)$, on a

$$
\begin{aligned}
\Theta(x, y, z) & =\frac{1}{2 i \pi} \int_{\beta-i L_{\varepsilon / 4}(z)}^{\beta+i L_{\varepsilon / 4}(z)} \frac{\zeta(s, y, z) x^{s}}{s} d s \\
+ & O_{\varepsilon}\left(\frac{x \exp \left\{-u \xi_{r}(u)+I_{r}\left(\xi_{r}(u)\right)-\min \left(c u,(\log y)^{3 / 5-\varepsilon}\right)\right\}}{(\log y) L_{\varepsilon}(z)}\right) .
\end{aligned}
$$


Lemme 8. Soit $\varepsilon>0$. Sous les conditions $\left(\mathrm{H}_{\varepsilon}\right)$ et $\left(\mathrm{V}_{\varepsilon}\right)$, on a

$$
\begin{aligned}
M(x, y, z)=\frac{x \zeta(1, y, z)}{2 i \pi} & \int_{-\xi-i L_{\varepsilon / 4}(z) \log y}^{-\xi+i L_{\varepsilon / 4}(z) \log y} \frac{e^{I_{r}(-s)}-r}{s+\log y} e^{u s} d s \\
& +O_{\varepsilon}\left(\frac{x \exp \left\{-u \xi_{r}(u)+I_{r}\left(\xi_{r}(u)\right)\right\}}{u(\log y) L_{\varepsilon}(z)}\right) .
\end{aligned}
$$

Lemme 9. Soit $\varepsilon>0$. Sous les conditions $\left(\mathrm{H}_{\varepsilon}\right)$ et $\left(\mathrm{V}_{\varepsilon}\right)$, on a

$$
\begin{aligned}
\int_{\beta-i L_{\varepsilon / 4}(z)}^{\beta+i L_{\varepsilon / 4}(z)} \frac{\zeta(s, y, z) x^{s}}{s} d s & \\
= & x \zeta(1, y, z) \int_{-\xi-i L_{\varepsilon / 4}(z) \log y}^{-\xi+i L_{\varepsilon / 4}(z) \log y} \frac{e^{I_{r}(-s)}-r}{s+\log y} e^{u s} d s \\
& +O_{\varepsilon}\left(\frac{x \exp \left\{-u \xi_{r}(u)+I_{r}\left(\xi_{r}(u)\right)\right\} \log 2 u}{\sqrt{u}(\log y) L_{\varepsilon}(z)}\right) .
\end{aligned}
$$

Ces formules étant triviales sous la condition $y \ll_{\varepsilon} 1$, on supposera dorénavant que $y \geq y_{0}(\varepsilon)$ où $y_{0}(\varepsilon)$ est une constante choisie suffisamment grande.

Démonstration du Lemme 7. On pose

$$
T=\max \left(L_{\varepsilon / 4}(z), L_{\varepsilon / 3}(z) \min \left(L_{\varepsilon / 2}(y), \exp \left\{u+(\log \log y)^{1+\varepsilon / 3}\right\}\right)\right) .
$$

En appliquant la formule de Perron sous une forme effective (voir par exemple le Théorème II 2.2 de [20]), on obtient

$$
\begin{aligned}
\Theta(x, y, z)- & \frac{1}{2 i \pi} \int_{\beta-i T}^{\beta+i T} \frac{\zeta(s, y, z) x^{s}}{s} d s \\
& \ll x^{\beta} \sum_{\substack{P^{-}(n)>z \\
P^{+}(n) \leq y}} \frac{n^{-\beta}}{1+T|\log (x / n)|} \\
& \ll \frac{x^{\beta} \zeta(\beta, y, z)}{\sqrt{T}}+\Theta\left(x+\frac{x}{\sqrt{T}}, y, z\right)-\Theta\left(x-\frac{x}{\sqrt{T}}, y, z\right) .
\end{aligned}
$$

Par (5.6), on voit que le premier terme de cette dernière expression est d'un ordre de grandeur convenable.

$\mathrm{Si}$

$$
u<(\log \log y)^{1+\varepsilon / 4}+(\log z)^{3 / 5-2 \varepsilon / 5},
$$


on a

$$
\begin{aligned}
R: & =\Theta\left(x+\frac{x}{\sqrt{T}}, y, z\right)-\Theta\left(x-\frac{x}{\sqrt{T}}, y, z\right) \ll \frac{x}{\sqrt{T}} \\
& \ll{ }_{\varepsilon} x \frac{\exp \left\{-u \xi_{r}(u)+I_{r}\left(\xi_{r}(u)\right)-u / 2\right\}}{(\log y) L_{\varepsilon / 2}(z)}
\end{aligned}
$$

sous la condition $\left(\mathrm{V}_{\varepsilon}\right)$, d'après le Lemme 1 . On suppose donc dorénavant

$$
u \geq(\log \log y)^{1+\varepsilon / 4}+(\log z)^{3 / 5-2 \varepsilon / 5} .
$$

En posant

$$
h(t)=\left|\frac{\sin (t \sqrt{T} / 2)}{t \sqrt{T} / 2}\right|^{2},
$$

on a

$$
R \ll \sum_{\substack{P^{-}(n)>z \\ P^{+}(n) \leq y}}\left(\frac{x}{n}\right)^{\beta} h\left(\log \left(\frac{x}{n}\right)\right)=\frac{1}{2 i \pi} \int_{\beta-i \infty}^{\beta+i \infty} \zeta(s, y, z) x^{s} \widehat{h}(s-\beta) d s .
$$

Comme

on a

$$
\widehat{h}(s)=\frac{1}{\sqrt{T}} \max (1-|s / \sqrt{T}|, 0),
$$

$$
R \ll \frac{1}{\sqrt{T}}\left|\int_{\substack{\kappa=\beta \\ T^{1 / 4} \leq|\tau| \leq \sqrt{T}}} \zeta(s, y, z) x^{s}\left(1-\frac{|\tau|}{\sqrt{T}}\right) d s\right|+\frac{x^{\beta} \zeta(\beta, y, z)}{T^{1 / 4}} .
$$

On majore le deuxième terme à l'aide de (5.6). Si $r \geq 1 / 2$, d'après le Lemme 6(i), le premier terme est

$$
\begin{aligned}
& \ll x^{\beta}\left(\frac{1}{\sqrt{T} \log x}+\frac{\log T}{\sqrt{T} \log y}+\frac{1}{L_{\varepsilon / 3}(z)}\right) \\
& \ll_{\varepsilon} \frac{x \exp \left\{-u \xi_{r}(u)+I_{r}\left(\xi_{r}(u)\right)-(\log y)^{3 / 5-\varepsilon}\right\}}{(\log y) L_{\varepsilon / 2}(z)}
\end{aligned}
$$

d'après le Lemme 1 et le Lemme 2(i). Si $r<1 / 2$, on majore l'intégrande à l'aide du Lemme 5. On obtient que le premier terme est

$$
\ll x^{\beta}(\log y)^{2} e^{I_{0}(r \xi)} \leq x \exp \left\{-u \xi+I_{r}(\xi)-u / 2\right\}(\log y)^{2},
$$

ce qui est ici convenable.

Pour conclure la démonstration du Lemme 7, il suffit maintenant de majorer convenablement

$$
I=\int_{\substack{\kappa=\beta \\ L_{\varepsilon / 4}(z) \leq|\tau| \leq T}} \frac{\zeta(s, y, z) x^{s}}{s} d s .
$$


Si l'on a $r>1 / 2$ ou

$$
\left\{u<(\log \log y)^{1+\varepsilon / 3}+(\log z)^{3 / 5-\varepsilon / 2} \text { et }(\log z)^{3 / 5-\varepsilon / 2}>\log \log y\right\},
$$

en choisissant $\varepsilon$ suffisamment petit et $y_{0}(\varepsilon)$ suffisamment grand, on voit que $T=L_{\varepsilon / 4}(z)$ et donc que $I=0$.

Si $u \geq(\log \log y)^{1+\varepsilon / 3}+(\log z)^{3 / 5-\varepsilon / 2}$ et $r \leq 1 / 2$, on majore $\zeta(s, y, z)$ par le Lemme 6(ii) et on obtient

$$
I \ll x^{\beta} e^{I_{r}(\xi)-c u}(\log y)^{2},
$$

ce qui est ici convenable.

Si enfin $u<(\log \log y)^{1+\varepsilon / 3}+(\log z)^{3 / 5-\varepsilon / 2}$ et $(\log z)^{3 / 5-\varepsilon / 2} \leq \log \log y$, on a par deux intégrations par parties successives,

$$
\begin{aligned}
I=\left[\frac{x^{s} \zeta(s, y, z)}{i s \log x}\right]_{\tau=L_{\varepsilon / 4}(z)}^{\tau=T}+\frac{1}{\log x} \int_{\substack{\kappa=\beta \\
L_{\varepsilon / 4}(z) \leq|\tau| \leq T}} \zeta(s, y, z) x^{s} \frac{d s}{s^{2}} \\
\\
\quad-\frac{1}{\log x} \int_{\substack{\kappa=\beta \\
L_{\varepsilon / 4}(z) \leq|\tau| \leq T}} \zeta^{\prime}(s, y, z) x^{s} \frac{d \tau}{s}
\end{aligned}
$$

et

$$
\begin{aligned}
& I=\left[\frac{x^{s} \zeta(s, y, z)}{i s \log x}+\frac{i x^{s} \zeta^{\prime}(s, y, z)}{s(\log x)^{2}}\right]_{\tau=L_{\varepsilon / 4}(z)}^{\tau=T}\left(\frac{\zeta(s, y, z)}{\log x}-\frac{\zeta^{\prime}(s, y, z)}{(\log x)^{2}}\right) \frac{x^{s}}{s^{2}} d s \\
&+ \int_{\substack{\kappa=\beta \\
L_{\varepsilon / 4}(z) \leq|\tau| \leq T}} \\
&+\int_{\substack{\kappa=\beta \\
L_{\varepsilon / 4}(z) \leq|\tau| \leq T}} \frac{\zeta^{\prime \prime}(s, y, z)}{(\log x)^{2}} x^{s} \frac{d \tau}{s} .
\end{aligned}
$$

On a par la formule de Cauchy

$$
\zeta^{(k)}(s, y, z)=k ! \int_{|w-s|=1 /(\log y)^{2 / 5+\varepsilon}} \frac{\zeta(w, y, z)}{(w-s)^{k+1}} d w .
$$

En majorant l'intégrande par l'intermédiaire du Lemme 5, on obtient que pour $L_{\varepsilon / 4}(z) \leq|\tau| \leq T$,

$$
\zeta^{(k)}(\beta+i \tau, y, z) \ll_{k, \varepsilon}(\log T)(\log z)(\log y)^{k(2 / 5+\varepsilon)} .
$$

En utilisant systématiquement ces majorations pour majorer $I$ par l'intermédiaire de la formule (6.4), on obtient

$$
I \ll x^{\beta}\left(\frac{1}{u(\log y) L_{\varepsilon / 4}(z)}+\frac{(\log T)^{2} \log z}{u^{2}(\log y)^{6 / 5-\varepsilon}}\right),
$$


ce qui est convenable en ayant pris soin de choisir $\varepsilon<1 / 5$. Cela conclut la démonstration du Lemme 7.

Démonstration du Lemme 8. Il faut d'abord établir la formule (4.2) qui résulte du lemme suivant:

Lemme 10. Soit

$$
\mu_{y, z}(u)=\int_{0}^{\infty} \sigma_{r}(u-t) y^{-t} d t
$$

de telle sorte que

$$
M(x, y, z)=x \prod_{z<p \leq y}\left(1-\frac{1}{p}\right)^{-1} \mu_{y, z}(u) .
$$

Alors on a sous les conditions $y>z \geq 1$ et $\operatorname{Re} s>-\log y$,

$$
\widehat{\mu}_{y, z}(s)=\frac{\exp \left\{I_{r}(-s)\right\}-r}{s+\log y} .
$$

Démonstration. Soit $\mathbf{1}(t)$ la fonction caractéristique de $\mathbb{R}_{+}$. D'après Friedlander ([6], Theorem $6(\mathrm{~A}))$, on a pour $0 \leq r \leq 1$ et $t \geq 1, \sigma_{r}(t) \ll_{r}$ $\varrho(t) \ll t^{-t}$. Cela montre que les transformées de Laplace des fonctions $\sigma_{r}(t)$ et $\mathrm{f}_{y}(t)=y^{-t} \mathbf{1}(t)$ convergent dans le demi-plan $\operatorname{Re} s>-\log y$. Comme $\mu_{y, z}$ est la convolée de ces deux fonctions, sa transformée de Laplace est le produit usuel des transformées. Or on a pour $\operatorname{Re} s>-\log y, \widehat{f}_{y}(s)=(s+\log y)^{-1}$ et $\widehat{\sigma}_{r}(s)=\exp \left\{I_{r}(-s)\right\}-r$ d'après le Lemme 1(iii) de [15]. Cela permet de conclure la démonstration du Lemme 10.

Revenons à la démonstration du Lemme 8 proprement dite. D'après le Lemme 10, en appliquant la formule d'inversion de Laplace on obtient sous la condition $\left(\mathrm{H}_{\varepsilon}\right)$

$$
M(x, y, z)=x \frac{\zeta(1, y, z)}{2 i \pi} \int_{-\xi-i \infty}^{-\xi+i \infty} \frac{e^{I_{r}(-s)}-r}{s+\log y} e^{u s} d s .
$$

Il suffit donc de majorer

$$
K=x \frac{\zeta(1, y, z)}{2 i \pi} \int_{\substack{\kappa=-\xi \\|\tau| \geq L_{\varepsilon / 4}(z) \log y}} \frac{e^{I_{r}(-s)}-r}{s+\log y} e^{u s} d s .
$$

On désigne par $K_{1}$ la contribution à $K$ correspondant au sous-domaine

$$
L_{\varepsilon / 4}(z) \log y \leq|\tau| \leq \max \left(L_{\varepsilon / 4}(z) \log y, e^{\xi}\right)=\eta
$$

et par $K_{2}$ la contribution à $K$ complémentaire. 
Par une intégration par parties, on a

$$
\begin{aligned}
& K_{2}=-\frac{x \zeta(1, y, z)}{i u} {\left[\frac{e^{u s}\left(e^{I_{r}(-s)}-r\right)}{s+\log y}\right]_{|\tau|=\eta}^{|\tau|=+\infty} } \\
&+\frac{x \zeta(1, y, z)}{i u} \int_{\substack{\kappa=-\xi \\
|\tau| \geq \eta}} \frac{e^{I_{r}(-s)+u s}}{s+\log y}\left(\frac{1}{s+\log y}+\frac{e^{-s}-e^{-r s}}{s}\right) d s .
\end{aligned}
$$

En appliquant le Lemme 2(v) pour estimer le terme tout intégré et majorer l'intégrale en valeur absolue, on obtient

$$
K_{2} \ll \frac{x e^{-u \xi+\xi}}{u(\log y) L_{\varepsilon / 4}(z)} \ll_{\varepsilon} \frac{x e^{-u \xi+I_{r}(\xi)}}{u(\log y) L_{\varepsilon}(z)}
$$

d'après le Lemme 1 et le Lemme 2(i).

Pour majorer $K_{1}$, on peut supposer

$$
e^{\xi}>L_{\varepsilon / 4}(z) \log y
$$

puisque sinon $K_{1}=0$. On désigne par $K_{1,1}$ la quantité

$$
K_{1,1}=-x \zeta(1, y, z) r \int_{\substack{\kappa=-\xi \\ L_{\varepsilon / 4}(z) \log y \leq|\tau| \leq e^{\xi}}} \frac{e^{u s}}{s+\log y} d s
$$

et par $K_{1,2}$ la quantité complémentaire de telle sorte que

$$
K_{1}=K_{1,1}+K_{1,2} .
$$

Pour majorer $K_{1,1}$, on déplace la droite d'intégration $\kappa=-\xi$ vers la gauche jusqu'à $-(\log y) / 2$ et on applique le théorème des résidus. On a

$$
\int_{\substack{\kappa=-(\log y) / 2 \\ L_{\varepsilon / 4}(z) \log y \leq|\tau| \leq e^{\xi}}} \frac{e^{u s}}{s+\log y} d s \ll \frac{e^{\xi}}{\sqrt{x}}
$$

et l'intégrale de $e^{u s} /(s+\log y)$ sur les bords horizontaux du rectangle est

$$
\ll \frac{e^{-u \xi}}{u \log y} \ll \frac{e^{-u \xi+\xi}}{u(\log y) L_{\varepsilon / 4}(z)}
$$

d'après (6.5). Comme il n'y a pas de résidu dans le rectangle en question, ces majorations démontrent que $K_{1,1}$ est d'un ordre de grandeur convenable.

En utilisant le Lemme 1 pour estimer $\xi$, on voit que sous les conditions $\left(\mathrm{H}_{\varepsilon}\right),\left(\mathrm{V}_{\varepsilon}\right)$ et $y \geq y_{0}(\varepsilon)$ avec $y_{0}(\varepsilon)$ choisi convenablement, la formule (6.5) entraîne que $r \leq 1 / 2$ et $(\log y) L_{\varepsilon / 4}(z) \leq e^{\xi} \ll u \log 2 u$. On a donc en 
utilisant le Lemme 2(iii)

$$
\begin{aligned}
K_{1,2} & =x \zeta(1, y, z) \quad \int_{\substack{\kappa=-\xi \\
L_{\varepsilon / 4}(z) \log y \leq|\tau| \leq e^{\xi}}} \frac{e^{I_{r}(-s)+u s}}{s+\log y} d s \\
& \ll x e^{-u \xi+I_{r}(\xi)-\sqrt{u}+\xi} \ll \frac{x e^{-u \xi+I_{r}(\xi)}}{u(\log y) L_{\varepsilon}(z)},
\end{aligned}
$$

ce qui permet d'achever la démonstration du Lemme 8.

Démonstration du Lemme 9. Soit $\varepsilon>0$ fixé. D'après le Lemme 1 , on a sous les conditions $\left(\mathrm{H}_{\varepsilon}\right)$ et $\left(\mathrm{V}_{\varepsilon}\right)$,

$$
\beta \geq 1-(\log y)^{-2 / 5-\varepsilon} .
$$

On peut donc appliquer le Lemme 4 avec $\kappa=\beta$. En effectuant le changement de variable $s^{\prime}=(s-1) \log y$ et en utilisant la formule de Mertens pour majorer $\zeta(1, y, z)$, on obtient donc

$$
\begin{aligned}
& \int_{\beta-i L_{\varepsilon / 4}(z)}^{\beta+i L_{\varepsilon / 4}(z)} \frac{\zeta(s, y, z) x^{s}}{s} d s \\
& -x \zeta(1, y, z) \int_{\ll_{\varepsilon}|J|+\frac{x}{L_{\varepsilon / 5}(y)} \int_{\substack{\kappa=-\xi \\
|\tau| \leq L_{\varepsilon / 4}(z) \log y}}^{-\xi+i L_{\varepsilon / 4}(z) \log y} \frac{e^{I_{r}(-s)}-r}{s+\log y} e^{u s} d s}\left|\exp \left\{I_{r}(-s)+u s\right\}\right||d s| \\
& +x\left|\int_{\substack{\kappa=-\xi \\
|\tau| \leq L_{\varepsilon / 4}(z) \log y}} \frac{e^{u s}}{s+\log y} d s\right|
\end{aligned}
$$

avec

$$
J=x \frac{\log y}{\log z} \int_{\substack{\kappa=-\xi \\|\tau| \leq L_{\varepsilon / 4}(z) \log y}} \exp \left\{I_{r}(-s)+u s\right\} \frac{F(1+s / \log y, z)}{s+\log y} d s .
$$

On majore le troisième terme de l'expression de droite de (6.6) comme on a majoré $K_{1,1}$ dans la démonstration du Lemme 8. On obtient que ce troisième terme est

$$
\ll_{\varepsilon} \frac{x e^{-u \xi}}{u(\log y) L_{\varepsilon / 3}(z)},
$$

ce qui est convenable. 
Le deuxième terme est trivialement

$$
\ll_{\varepsilon} x e^{-u \xi+I_{r}(\xi)} \frac{L_{\varepsilon / 4}(z) \log y}{L_{\varepsilon / 5}(y)} \ll_{\varepsilon} \frac{x e^{-u \xi+I_{r}(\xi)}}{L_{\varepsilon / 3}(y)},
$$

ce qui est également convenable.

Il reste à majorer $J$. Pour cela, on ne s'intéressera qu'au sous-domaine d'intégration correspondant aux $\tau$ positifs, le travail pour les $\tau$ négatifs étant en tout point semblable. On découpe l'intervalle $\left[0, L_{\varepsilon / 4}(z) \log y\right]$ en cinq sous-intervalles d'extrémités

$$
\begin{gathered}
0,1 / \sqrt{u}, 1, \min \left(L_{\varepsilon / 4}(z)(\log y), e^{\xi}\right), \\
\max \left(\min \left(L_{\varepsilon / 4}(z)(\log y), e^{\xi}\right),(2 r)^{-1}\right) \quad \text { et } \quad L_{\varepsilon / 4}(z)(\log y) .
\end{gathered}
$$

On désigne par $J_{1}, J_{2}, J_{3}, J_{4}$ et $J_{5}$ les contributions correspondantes.

Notons tout d'abord qu'en utilisant la formule de Cauchy

$$
F^{(k)}(s, z)=k ! \int_{|w-s|=(\log z)^{-1}} \frac{F(w, z)}{(w-s)^{k+1}} d w,
$$

la deuxième partie du Lemme 4 permet de montrer que

$$
F^{(k)}(\beta+i \tau, z) \ll_{\varepsilon} \frac{k !(\log z)^{k}}{L_{\varepsilon / 4}(z)} \quad\left(|\tau| \ll L_{\varepsilon / 4}(z)\right) .
$$

Pour majorer $J_{5}$, on peut supposer que

$$
e^{\xi}<L_{\varepsilon / 4}(z) \log y
$$

puisque sinon $J_{5}=0$. On redécoupe l'intervalle d'intégration de $J_{5}$ en intervalles $\left[\tau_{j}, \tau_{j+1}\right]\left(j_{0} \leq j<j_{1}\right)$ avec

$$
\begin{aligned}
j_{0} & =\max \left(1,\left[2 r e^{\xi}\right]\right), \quad j_{1} & =\left[2 r L_{\varepsilon / 4}(z) \log y\right], \\
\tau_{j_{0}} & =\max \left(e^{\xi},(2 r)^{-1}\right), \quad \tau_{j_{1}} & =L_{\varepsilon / 4}(z) \log y
\end{aligned}
$$

et

$$
\tau_{j}=(2 r)^{-1} j \quad\left(j_{0}<j<j_{1}\right) .
$$

Dans chaque intervalle $\left[\tau_{j}, \tau_{j+1}\right]$, on développe $F$ en série entière autour de $s=\tau_{j}$. On obtient en posant $s_{j}=\beta+i \tau_{j} / \log y$

$$
\begin{aligned}
J_{5}= & x \frac{\log y}{\log z} \sum_{j=j_{0}}^{j_{1}-1} \sum_{k=0}^{\infty} \frac{i^{k+1} F^{(k)}\left(s_{j}, z\right)}{k !(\log y)^{k}} \\
& \times \int_{\substack{\kappa=-\xi \\
\tau_{j} \leq \tau \leq \tau_{j+1}}} \frac{e^{I_{r}(-s)+u s}}{s+\log y}\left(\tau-\tau_{j}\right)^{k} d \tau .
\end{aligned}
$$


Par une intégration par parties, l'intégrale est

$$
\begin{aligned}
& \frac{1}{i u}\left[\frac{e^{I_{r}(-s)+u s}}{s+\log y}\left(\tau-\tau_{j}\right)^{k}\right]_{\tau=\tau_{j}}^{\tau=\tau_{j+1}} \\
&+\frac{1}{u} \int_{\substack{\kappa=-\xi \\
\tau_{j} \leq \tau \leq \tau_{j+1}}} e^{I_{r}(-s)+u s}\left[\frac{\left(\tau-\tau_{j}\right)^{k}}{(s+\log y)^{2}}+\frac{i k\left(\tau-\tau_{j}\right)^{k-1}}{s+\log y}\right. \\
&\left.+\frac{\left(\tau-\tau_{j}\right)^{k}\left(e^{-s}-e^{-r s}\right)}{i s(s+\log y)}\right] d \tau .
\end{aligned}
$$

Par le Lemme $2(\mathrm{v})$, on a ici $e^{I_{r}(-s)+u s} \ll r e^{-u \xi}$. De plus, pour $m \geq 0$, $n_{1} \geq 0, n_{2} \geq 0$ et $n_{1}+n_{2} \geq 1$, on a

$$
\begin{aligned}
\int_{\tau_{j}}^{\tau_{j+1}}\left|\frac{\left(\tau-\tau_{j}\right)^{m}}{s^{n_{1}}(s+\log y)^{n_{2}}}\right| d \tau & \ll \frac{1}{\tau_{j}^{n_{1}+n_{2}}} \int_{\tau_{j}}^{\tau_{j+1}}\left(\tau-\tau_{j}\right)^{m} d \tau \\
& \ll \frac{(2 r)^{n_{1}+n_{2}-m-1}}{j(m+1)} .
\end{aligned}
$$

Cela permet de montrer que l'expression (6.9) est

$$
\ll(2 r)^{2-k} e^{-u \xi+\xi} j^{-1} .
$$

En substituant dans (6.8) et en utilisant (6.7), on obtient alors

$$
J_{5} \ll_{\varepsilon} \frac{x r e^{-u \xi+\xi}}{L_{\varepsilon / 4}(z)} \sum_{j=1}^{j_{1}} \sum_{k=0}^{\infty} \frac{1}{j 2^{k}} \ll_{\varepsilon} \frac{x e^{-u \xi+\xi}}{(\log y) L_{\varepsilon / 2}(z)},
$$

ce qui est convenable.

Pour majorer $J_{4}$, on peut supposer que

$$
2 r e^{\xi}<1 \quad \text { et } \quad e^{\xi}<L_{\varepsilon / 4}(z) \log y
$$

puisque sinon $J_{4}=0$. Le domaine d'intégration de $J_{4}$ est alors $\left[e^{\xi},(2 r)^{-1}\right]$. Comme pour $J_{5}$, on développe $F$ en série entière, cette fois autour de 1 , et on intègre par parties. On obtient

$$
J_{4}=x \frac{\log y}{\log z} \sum_{k=1}^{\infty} \frac{F^{(k)}(1, z)}{k !(\log y)^{k}} \int_{\substack{\kappa=-\xi \\ e^{\xi} \leq \tau \leq(2 r)^{-1}}} \frac{e^{I_{r}(-s)+u s} s^{k}}{s+\log y} d s
$$

avec l'intégrale qui est 


$$
\begin{aligned}
\frac{1}{i u}\left[\frac{e^{I_{r}(-s)+u s}}{s+\log y} s^{k}\right]_{\tau=e^{\xi}}^{\tau=(2 r)^{-1}} & \\
-\frac{e^{-\gamma}}{i u} \int_{\substack{\kappa=-\xi \\
e^{\xi} \leq \tau \leq(2 r)^{-1}}} & \left(\frac{1}{s}+O\left(\frac{e^{\xi}}{\tau^{2}}+r\right)\right)\left[\frac{s^{k}}{(s+\log y)^{2}}\right. \\
& \left.+\frac{k s^{k-1}}{s+\log y}+\frac{s^{k}\left(e^{-s}-e^{-r s}\right)}{s(s+\log y)}\right] e^{u s} d s
\end{aligned}
$$

où on a utilisé le Lemme 2(iv) pour estimer $e^{I_{r}(-s)}$.

Pour cette dernière intégrale, on développe l'intégrande et on estime les termes de la forme

$$
\int_{\substack{\kappa=-\xi \\ e^{\xi} \leq \tau \leq(2 r)^{-1}}} \frac{s^{m}}{(s+\log y)^{n}} e^{(u-a) s} d s \quad(a=0, r \text { ou } 1)
$$

en déplaçant la droite d'intégration vers la gauche et en appliquant le théorème des résidus. De plus, on majore les termes de la forme

$$
\int_{\substack{\kappa=-\xi \\ e^{\xi} \leq \tau \leq(2 r)^{-1}}} O\left(\frac{s^{m}}{(s+\log y)^{n}} e^{(u-a) s}\right)|d s|
$$

par

$$
\frac{e^{-u \xi+\xi}}{\log y}(2 r)^{-m-1} .
$$

On montre ainsi que l'expression (6.11) est $\ll e^{-u \xi+\xi}(2 r)^{2-k}$. En substituant dans (6.10) et en utilisant (6.7), on obtient alors

$$
J_{4} \ll_{\varepsilon} \frac{x r e^{-u \xi+\xi}}{L_{\varepsilon / 4}(z)} \sum_{k=1}^{\infty} 2^{-k} \ll_{\varepsilon} \frac{x e^{-u \xi+\xi}}{(\log y) L_{\varepsilon / 2}(z)},
$$

ce qui est convenable.

Pour majorer $J_{3}, J_{2}$ et $J_{1}$, on majore l'intégrande en valeur absolue en utilisant systématiquement le Lemme 2 pour $\exp \left\{I_{r}(-s)+u s\right\}$ et (5.2) pour $F(1+s / \log y, z)$. Si $e^{\xi} \geq L_{\varepsilon / 3}(z)$, sous les conditions $\left(\mathrm{H}_{\varepsilon}\right),\left(\mathrm{V}_{\varepsilon}\right)$ et $y \geq y_{0}(\varepsilon)$ avec $y_{0}(\varepsilon)$ convenablement choisi, on a $r \leq 1 / 2$ et donc

$$
\begin{aligned}
J_{3} & \ll \varepsilon \frac{x e^{-u \xi+I_{r}(\xi)}}{u^{4} L_{\varepsilon / 4}(z)} \int_{\substack{\kappa=-\xi \\
1 \leq \tau \leq e^{\xi}}}\left|\frac{s}{s+\log y}\right| d s \mid \\
& \ll \frac{x e^{-u \xi+I_{r}(\xi)+2 \xi}}{u^{4}(\log y) L_{\varepsilon / 4}(z)} \ll \frac{x e^{-u \xi+I_{r}(\xi)}}{u(\log y) L_{\varepsilon / 4}(z)} .
\end{aligned}
$$


Si $e^{\xi}<L_{\varepsilon / 3}(z)$, on a

$$
\begin{aligned}
J_{3} & \ll \varepsilon \frac{x e^{-u \xi+I_{r}(\xi)}}{L_{\varepsilon / 4}(z)} \int_{\substack{\kappa=-\xi \\
1 \leq \tau \leq e^{\xi}}}\left|\frac{s}{s+\log y}\right||d s| \\
& \ll \frac{x e^{-u \xi+I_{r}(\xi)+2 \xi}}{(\log y) L_{\varepsilon / 4}(z)} \ll \frac{x e^{-u \xi+I_{r}(\xi)}}{u(\log y) L_{\varepsilon}(z)} .
\end{aligned}
$$

Pour $J_{2}$, on obtient

$$
\begin{aligned}
J_{2} & \ll \varepsilon \frac{x e^{-u \xi+I_{r}(\xi)}}{(\log y) L_{\varepsilon}(z)} \int_{\substack{\kappa=-\xi \\
u^{-1 / 2} \leq \tau \leq 1}} e^{-c \tau^{2} u}|s||d s| \\
& \ll \frac{x e^{-u \xi+I_{r}(\xi)}}{\sqrt{u}(\log y) L_{\varepsilon}(z)} \xi \ll \frac{x e^{-u \xi+I_{r}(\xi)} \log 2 u}{\sqrt{u}(\log y) L_{\varepsilon}(z)} .
\end{aligned}
$$

Enfin,

$$
J_{1} \ll \frac{x e^{-u \xi+I_{r}(\xi)}}{(\log y) L_{\varepsilon}(z)} \int_{\substack{\kappa=-\xi \\ 0 \leq \tau \leq u^{-1 / 2}}}|s||d s| \ll \varepsilon \frac{x e^{-u \xi+I_{r}(\xi)} \log 2 u}{\sqrt{u}(\log y) L_{\varepsilon}(z)},
$$

ce qui achève la démonstration du Lemme 9.

\section{Bibliographie}

[1] N. G. de Bruijn, On the number of uncancelled elements in the sieve of Eratosthenes, Nederl. Akad. Wetensch. Proc. 53 (1950), 803-812.

[2] - On the number of positive integers $\leq x$ and free of prime factors $>y$, ibid. 54 (1951), 50-60.

[3] E. R. Canfield, P. Erdős and C. Pomerance, On a problem of Oppenheim concerning "Factorisatio Numerorum", J. Number Theory 17 (1983), 1-28.

[4] P. Erdős et G. Tenenbaum, Sur les densités de certaines suites d'entiers, Proc. London Math. Soc. (3) 59 (1989), 417-438.

[5] E. Fouvry et G. Tenen baum, Entiers sans grand facteur premier en progressions arithmétiques, ibid. 63 (1991), 449-494.

[6] J. B. Friedlander, Integers free from large and small primes, ibid. 33 (1976), $565-576$.

[7] J. B. Friedlander, A. Granville, A. Hildebrand and H. Maier, Oscillation theorems for primes in arithmetic progressions and for sifting functions, J. Amer. Math. Soc. 4 (1991), 25-86.

[8] A. Hildebrand, On the number of positive integers $\leq x$ and free of prime factors $>y$, J. Number Theory 22 (1986), 289-307.

[9] - On the number of prime factors of integers without large prime divisors, ibid. 25 (1987), 81-106.

[10] A. Hildebrand and H. Maier, Irregularities in the distribution of primes in short intervals, J. Reine Angew. Math. 397 (1989), 162-193. 
[11] A. Hildebrand and G. Tenenbaum, On integers free of large prime factors, Trans. Amer. Math. Soc. 296 (1986), 265-290.

[12] - - - On the number of prime factors of an integer, Duke Math. J. 56 (3) (1988), 471-501.

[13] B. V. Levin and A. S. Faŭnleı̆b, Applications of some integral equations to problems in number theory, Russian Math. Surveys 22 (3) (1967), 119-204.

[14] E. Saias, Sur le nombre des entiers sans grand facteur premier, J. Number Theory 32 (1989), 78-99.

[15] -, Entiers sans grand ni petit facteur premier I, Acta Arith. 61 (1992), 347-374.

[16] —, Entiers sans grand ni petit facteur premier III, prépublication.

[17] H. Smida, Valeur moyenne des fonctions de Piltz sur les entiers sans grand facteur premier, ce volume, 21-50.

[18] G. Tenenbaum, Sur un problème extrémal en arithmétique, Ann. Inst. Fourier (Grenoble) 37 (2) (1987), 1-18.

[19] —, La méthode du col en théorie analytique des nombres, dans : Séminaire de Théorie des Nombres, Paris 1986-87, C. Goldstein (ed.), Progr. Math. 75, Birkhäuser, 1988, 411-442.

[20] —, Introduction à la théorie analytique et probabiliste des nombres, Publ. Institut Elie Cartan 13, Université de Nancy I, 1990.

LABORATOIRE DE PROBABILITÉS

UNIVERSITÉ PARIS VI

4, PLACE JUSSIEU

75252 PARIS CEDEX 05, FRANCE 